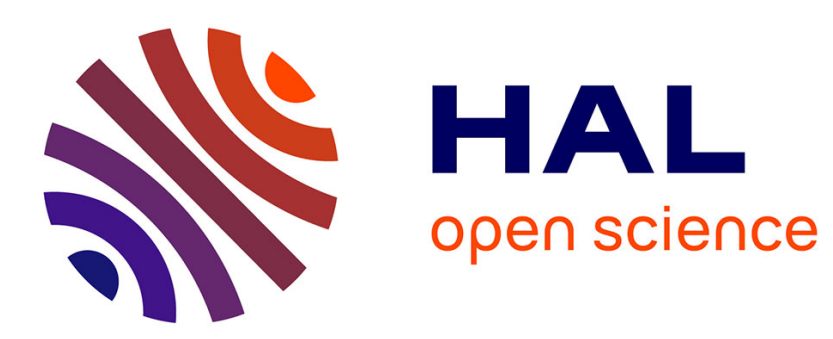

\title{
An Instrumental Variable Approach for Aircraft Identification
}

Mathieu Brunot

\section{To cite this version:}

Mathieu Brunot. An Instrumental Variable Approach for Aircraft Identification. AIAA Scitech 2020

Forum, Jan 2020, Orlando, United States. 10.2514/6.2020-0509 . hal-03232425

\section{HAL Id: hal-03232425 \\ https://hal.science/hal-03232425}

Submitted on 21 May 2021

HAL is a multi-disciplinary open access archive for the deposit and dissemination of scientific research documents, whether they are published or not. The documents may come from teaching and research institutions in France or abroad, or from public or private research centers.
L'archive ouverte pluridisciplinaire HAL, est destinée au dépôt et à la diffusion de documents scientifiques de niveau recherche, publiés ou non, émanant des établissements d'enseignement et de recherche français ou étrangers, des laboratoires publics ou privés. 


\title{
An Instrumental Variable Approach for Aircraft Identification
}

\author{
Mathieu Brunot* \\ ONERA, Toulouse, FRANCE
}

\section{Nomenclature}

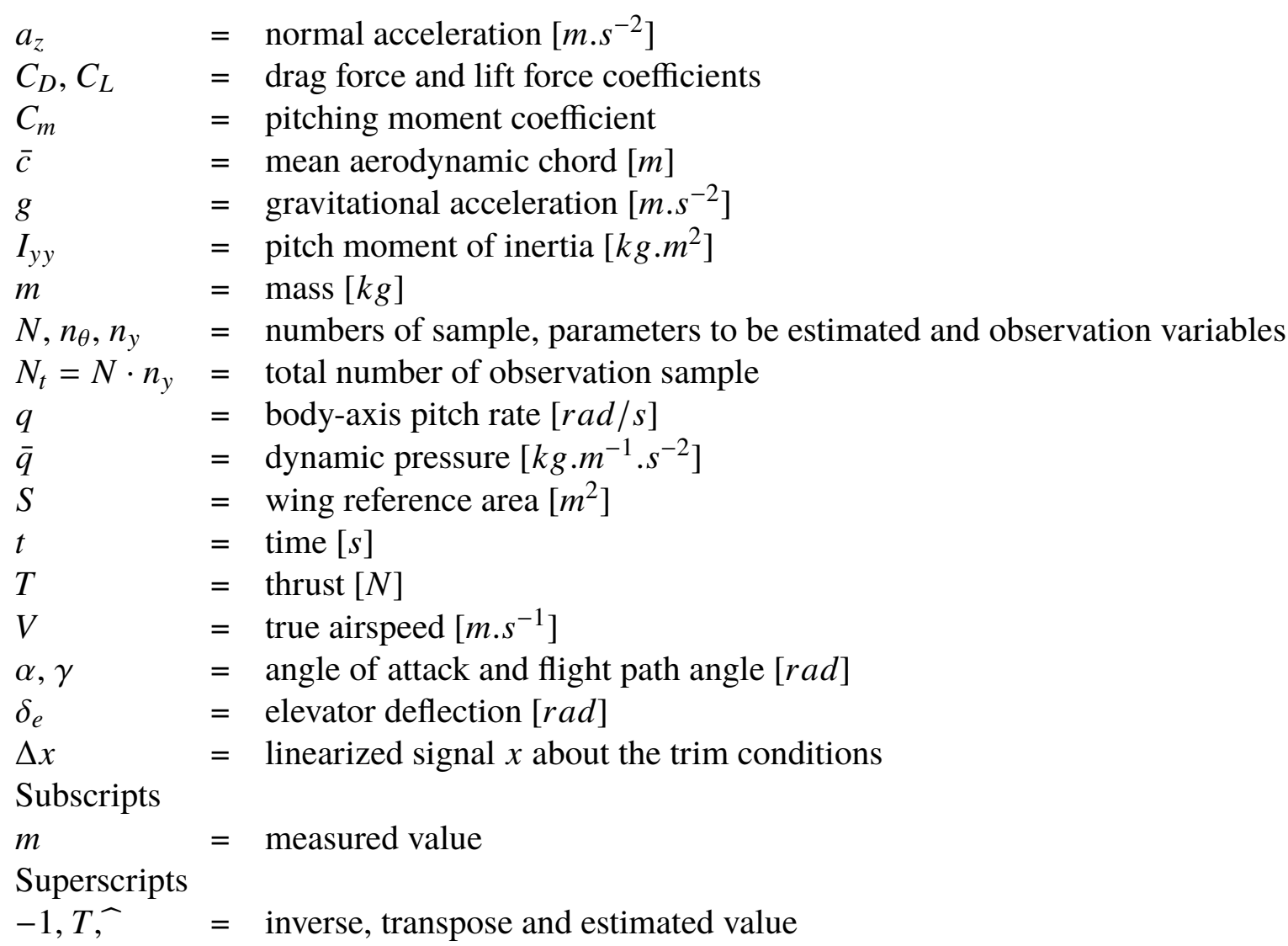

\section{Introduction}

$\mathrm{T}$ HE equation error approach is widely used in many fields and was one the first employed techniques for aircraft dynamic model identification [1]. That approach is usually based on the Least-Squares (LS) optimization that allows estimating stability and control derivatives from small-perturbation maneuvers about a trimmed flight condition. The LS method has the advantage to be easy to implement and allows a straightforward analysis of the modeling results. However, the major limitation of this method is that it provides asymptotically biased and inconsistent

*Research Engineer, DTIS, mathieu.brunot@ onera.fr. 
estimated parameters in the case of measurement errors and noise in the independent variables [2]. Furthermore, an unmeasured excitation like turbulence can contaminate the dynamical model. To minimize these errors, high-quality sensors can be used on the one hand, and on the other, specific preprocessing techniques can be employed. In fact, techniques have been developed over the years to improve the effectiveness of the LS method by applying specific smoothing processes and using frequency-domain data [3, 4].

As long as possible, stability and control flight testing should be conducted in smooth air to avoid the adverse effects of turbulence. Nonetheless, such a strategy is not always possible due to the constraints of the aircraft flight domain, e.g. a subscale aircraft [5]. Two strategies can be considered to deal with the turbulence for the aircraft model identification. Firstly, an analytic model of the turbulence can be designed and incorporated into the aircraft dynamic model. For instance, the Filter Error Method (FEM) [6] relies on a Kalman filter that estimate the aircraft states with stochastic process noises modeling the turbulence. The combination of the Kalman filter with the nonlinear optimization algorithm may make the overall process difficult to employ in practice. Secondly, turbulence can be eliminated or marginalized by a dedicated signal preprocessing combined with an appropriate estimation method. The equation error approach in the frequency-domain proved to be well-suited for this task, after a careful selection of the frequency-domain data [5].

The Instrumental Variable (IV) technique was introduced by Reiersøl [7] to avoid the difficulties of independent variables correlated with the errors. The basic idea is to introduce a set of instruments correlated with independent variables but the errors. Many variants exist depending on the way in which the instruments are generated. This technique leads to consistent estimated parameters while relying on simple linear regression algorithms, which can explained its attractiveness for the automatic control community [8-10]. Recently, the technique was adapted to identify electromechanical systems such as industrial robots [11]. The instruments are generated with the simulation of the robot closed-loop model. Regarding aircraft applications, the contribution of Klein [2] must be cited, where the instruments are generated via a Kalman filter. In Ref. [12], the authors considered the sequential identification of a longitudinal aerodynamic model via an IV technique in the frequency domain.

In this paper, a generic IV approach devoted to aircraft identification in time domain is introduced. In fact, two variants depending on the preprocessing are suggested. The instrument set comes from the identification model using the simulated data of the flight dynamics model. That simulation requires the same input signals as the studied aircraft and the estimated parameters from the previous iteration. The two variants distinguish themselves through the differentiation of the measured signals necessary for the identification. The first one relies on finite differences coupled with a low-pass filter tuned by the user. The second one is based on the filtering through the transfer function of the dynamic model identified at the previous iteration. Thus, the process iteratively selects the bandwidth of interest while providing the derivatives. The resulting algorithms improve the noise immunity of the estimated parameters with respect to corrupted data in the observation matrix.

The remainder of this paper is organized as follows. Section II reviews the usual longitudinal flight dynamic model and the Least-Squares identification technique. Section III is devoted to the IV approaches for aircraft identification. The suggested approaches are evaluated via Monte Carlo simulations with the Generic Transport Model (GTM) in Section IV] Finally, Section V provides concluding remarks. 


\section{Problem Description}

\section{A. Longitudinal Dynamic Model}

In this article, we restrict ourselves to the longitudinal motion of an aircraft assuming the aircraft is symmetric. With respect to Ref. [13] for instance, the nonlinear dynamics (without navigation equations) in the wind axes is given by:

$$
\begin{aligned}
m \dot{V} & =-\bar{q} S C_{D}+T \cos (\alpha)-m g \sin (\gamma) & I_{y y} \dot{q} & =\bar{q} S \bar{c} C_{m} \\
m V \dot{\alpha} & =-\bar{q} S C_{L}+q+m g \cos (\gamma)-T \sin (\alpha) & q & =\dot{\alpha}+\dot{\gamma}
\end{aligned}
$$

If that nonlinear model is considered for the simulation to generate flight test data, the system to be identified is here the Short-Period Pitch Oscillation (SPPO). The linearized and reduced order model is:

$$
\begin{aligned}
{\left[\begin{array}{c}
\Delta \dot{\alpha}(t) \\
\dot{q}(t)
\end{array}\right] } & =\left[\begin{array}{cc}
-\frac{\bar{q} S}{m V} C_{L \alpha} & 1-\frac{\bar{q} S}{m V} \frac{\bar{c}}{2 V} C_{L q} \\
\frac{\bar{q} S \bar{C}}{I_{y y}} C_{m \alpha} & \bar{q} S \bar{c} \frac{\bar{c}}{I_{y y}} C_{m q}
\end{array}\right]\left[\begin{array}{c}
\Delta \alpha(t) \\
q(t)
\end{array}\right]+\left[\begin{array}{c}
-\frac{\bar{q} S}{m V} C_{L \delta_{e}} \\
\frac{\bar{q} S \bar{c}}{I_{y y}} C_{m \delta_{e}}
\end{array}\right] \Delta \delta_{e}(t) \\
{\left[\begin{array}{c}
\Delta \alpha_{m}(t) \\
q_{m}(t) \\
\Delta a_{z_{m}}(t)
\end{array}\right] } & =\left[\begin{array}{cc}
1 & 0 \\
0 & 1 \\
-\frac{\bar{q} S}{m g} C_{L \alpha} & -\frac{\bar{q} S}{m g} \frac{\bar{c}}{2 V} C_{L q}
\end{array}\right]\left[\begin{array}{c}
\Delta \alpha(t) \\
q(t)
\end{array}\right]+\left[\begin{array}{c}
0 \\
0 \\
-\frac{\bar{q} S}{m g} C_{L \delta_{e}}
\end{array}\right] \Delta \delta_{e}(t)+\left[\begin{array}{c}
v_{\alpha}(t) \\
v_{q}(t) \\
v_{a_{z}}(t)
\end{array}\right],
\end{aligned}
$$

where $\Delta x=x-x_{0}$ with $x_{0}$ the trimmed value of $x=\alpha, a_{z}$ or $\delta_{e}$. We assume that at the trim conditions $q=\dot{q}=0$. Hence, the $\Delta$ is dropped for the corresponding linearized signals.

\section{B. SPPO Identification Model}

From the linearized model, a formulation linear with respect to the stability and control derivatives to be estimated can be derived:

$$
\boldsymbol{y}(t)=\left[\begin{array}{lll}
\Delta \dot{\alpha}(t)-q_{m}(t) & \dot{q}(t) & \Delta a_{z_{m}}(t)
\end{array}\right]^{T}=\boldsymbol{X}(t) \boldsymbol{\theta}+\boldsymbol{v}(t)
$$

where

- $\boldsymbol{\theta}=\left[\begin{array}{llllll}C_{L \alpha} & C_{L q} & C_{L \delta_{e}} & C_{m \alpha} & C_{m q} & C_{m \delta_{e}}\end{array}\right]^{T}$ is a $\left(n_{\theta} \times 1\right)$ vector of unknown parameters;

- $\boldsymbol{v}(t)=\left[\begin{array}{lll}v_{1}(t) & v_{2}(t) & v_{3}(t)\end{array}\right]^{T}$ is a $\left(n_{y} \times 1\right)$ vector of error terms;

- $\boldsymbol{X}(t)=\left[\begin{array}{cccccc}\frac{1}{m V} X_{\alpha}(t) & \frac{1}{m V} X_{q}(t) & \frac{1}{m V} X_{\delta_{e}}(t) & 0 & 0 & 0 \\ 0 & 0 & 0 & -\frac{\bar{c}}{I_{y y}} X_{\alpha}(t) & -\frac{\bar{c}}{I_{y y}} X_{q}(t) & -\frac{\bar{c}}{I_{y y}} X_{\delta_{e}}(t) \\ \frac{1}{m g} X_{\alpha}(t) & \frac{1}{m g} X_{q}(t) & \frac{1}{m g} X_{\delta_{e}}(t) & 0 & 0 & 0\end{array}\right]$ is the $\left(n_{y} \times n_{\theta}\right)$ observation matrix built with the independent variables $X_{\alpha}(t)=-\bar{q} S \Delta \alpha(t), X_{q}(t)=-\bar{q} S \frac{\bar{c}}{2 V} q(t)$ and $X_{\delta_{e}}(t)=-\bar{q} S \Delta \delta_{e}(t)$.

It is assumed that $\boldsymbol{X}$ is full column rank, i.e. $\operatorname{rank}(\boldsymbol{X})=n_{\theta}=6$, and that $N_{t}=N \cdot n_{y} \gg n_{\theta}$, to have an over-determined system of equations. Based on (4), the parameters can be estimated 
straightforwardly with the LS method [13]:

$$
\widehat{\boldsymbol{\theta}}_{L S}(N)=\left[\frac{1}{N} \sum_{i=1}^{N} \boldsymbol{X}^{T}\left(t_{i}\right) \boldsymbol{X}\left(t_{i}\right)\right]^{-1}\left[\frac{1}{N} \sum_{i=1}^{N} \boldsymbol{X}^{T}\left(t_{i}\right) \boldsymbol{y}\left(t_{i}\right)\right] .
$$

With (4), it appears the need to evaluate the derivatives $\dot{\alpha}$ and $\dot{q}$ from the measurements. Angular accelerations are usually not measured, or else with poor quality. The preprocessing step must be accurately done in order to limit the distortions of the signals.

\section{Limitations of the Least-Squares Estimation}

From a theoretical point of view, the LS estimates (5) are unbiased if

- the error is stationary random vector with zero mean: $\mathrm{E}[v]=0$,

- the error is uncorrelated with the independent variables: $\mathrm{E}\left[\boldsymbol{X}^{T} \boldsymbol{v}\right]=\mathrm{E}\left[\boldsymbol{X}^{T}\right] \mathrm{E}[\boldsymbol{v}]=0$,

where $E[\cdot]$ denotes the mathematical expectation. In practice, the measured states and input variables are corrupted by errors. Thus, the observation vector $\boldsymbol{y}$ and the observation matrix $\boldsymbol{X}$ are polluted by noises; see e.g. [2]. When the independent variables are measured with errors, the situation is usually referred to as errors in variables. That causes the error terms to be correlated with the noisy independent variables. Consequently, the LS are biased.

\section{Instrumental Variable Estimator}

\section{A. Principles of the Instrumental Variable Method}

In the case of the IV approach, an $\left(n_{y} \times n_{\theta}\right)$ instrumental matrix, denoted by $\boldsymbol{Z}$, is introduced that must fulfill the following conditions:

- $\mathrm{E}\left[\boldsymbol{Z}^{T} \boldsymbol{X}\right]$ is full column rank,

- $\mathrm{E}\left[\boldsymbol{Z}^{T} \boldsymbol{v}\right]=0$.

The first condition means that the instrumental matrix must be well correlated with the observations and is sometimes termed the instrument relevance [14]. The second condition expresses the fact that the instrumental matrix must be uncorrelated with the error and is sometimes known as the instrument exogeneity. Assuming that the two previous conditions hold, it can be shown that the IV parameter estimates $\widehat{\boldsymbol{\theta}}_{I V}(N)$ given by

$$
\widehat{\boldsymbol{\theta}}_{I V}(N)=\left[\frac{1}{N} \sum_{i=1}^{N} \boldsymbol{Z}^{T}\left(t_{i}\right) \boldsymbol{X}\left(t_{i}\right)\right]^{-1}\left[\frac{1}{N} \boldsymbol{Z}^{T}\left(t_{i}\right) \boldsymbol{y}\left(t_{i}\right)\right],
$$

are asymptotically unbiased and consistent. Note that the standard LS solution is obtained if $\boldsymbol{Z}$ is replaced by $\boldsymbol{X}$ but, as explained in the previous section, the resulting estimates will be asymptotically biased and inconsistent, in general due to the noise on the data.

One key feature of the IV method is the construction of the instruments; i.e. the elements of the instrumental matrix. For example, a valid instrumental matrix is $\boldsymbol{Z}=\boldsymbol{X}_{n f}$, assuming that the observation matrix can be decomposed such as $\boldsymbol{X}=\boldsymbol{X}_{n f}+\boldsymbol{V}$, where $\boldsymbol{V}$ is a $\left(n_{y} \times n_{\theta}\right)$ matrix of error terms uncorrelated with the noise-free one: $\boldsymbol{X}_{n f}$. That linear decomposition of the observation matrix is valid for the SPPO model (4) because it is linear with respect to noisy signals $\Delta \alpha, q$ and 
$\Delta \delta_{e}$. Consequently, we have $\mathrm{E}\left[\boldsymbol{Z}^{T} \boldsymbol{X}\right]=\mathrm{E}\left[\boldsymbol{X}_{n f}^{T} \boldsymbol{X}_{n f}\right]$ that is symmetric. As explained in Example 3.2 in Ref. [9], that matrix is positive definite if the model is not over-parametrized and if the input is exciting enough. For instrument exogeneity, it comes $\mathrm{E}\left[\boldsymbol{Z}^{T} \boldsymbol{v}\right]=\mathrm{E}\left[\boldsymbol{X}_{n f}^{T} \boldsymbol{v}\right]=0$. The condition for this last relation is that the input generating the instruments and the error $v$ are independent. Therefore, the objective is to construct an instrumental matrix that tends towards the noise-free part of the observation matrix, which is of course not known.

\section{B. Instrument Generation}

In automatic control, several IV solutions have been developed for different configurations, especially for closed-loop identification [10]. Instruments can be constructed in many ways [9], but one of the most successful ways is to use an auxiliary model of the system [15]. Assuming the input signal is perfectly known (i.e. noise free), the simulation provides noise free signals to construct the instrumental matrix using the same equations as those of the observation matrix. The noise free aspect ensures instrument exogeneity. Regarding the instrument relevance, it is based on the assumptions that the parameters used for the auxiliary model are reasonable and that there is no modeling error. Since the auxiliary model requires the parameters, this IV approach is an iterative process: parameters from the previous iteration are used for the auxiliary model on the next iteration. For aircraft identification, two solutions can be considered for the auxiliary model. The practitioner can either simulate the nonlinear model (1) or just the linearized one about the trim conditions (2). The latter seems more appropriate to estimate the parameters since it relies on the same assumptions as the identified aerodynamic model and it has a lower computational cost than the nonlinear one. However, the user should be cautious with the input signal. If it is a measured surface deflection, it should appropriately be filtered to avoid noise correlation to ensure instrument exogeneity. In the case of an aircraft operating in closed-loop, it would be more interesting to use the reference signal and simulate the whole closed-loop system, just as robot identification [11].

\section{Differentiating Noisy Time Signals}

One element not mentioned in section $\Pi$ II. is the need of differentiating the measured signals to get the derivatives of $\alpha$ and $q$ for the observation vector. In the case of unsteady aerodynamics, those derivatives may even be included in the observation matrix, although it is not without risks [16]. Several methodologies to estimate the derivatives are proposed in Ref. [13], but it has been recently highlighted that an appropriate low-pass filtering in combination with finite differences performs very well [17]. The idea is to design a low-pass filter that preserves the system's bandwidth (unitary gain and zero lag) while rejecting the noise. Typically, a Butterworth filter is employed in both forward and backward directions to avoid any lag distortion.

Inspired by the Simplified Refined Instrumental Variable for Continuous-time systems (SRIVC) [15], one can think about filtering the measured signals by the system's dynamics:

$$
f_{j}^{1}(p)=\frac{p^{j}}{\Delta_{d y n}(p)}=\frac{p^{j}}{|p \boldsymbol{I}-\boldsymbol{A}|},
$$

where $\boldsymbol{A}$ is the state matrix of the linearized model (2) and $p=\frac{d}{d t}$ is the differential operator; see e.g. [18]. In the case of the reduced order model of the SPPO, $\Delta_{d y n}(p)$ is a second order polynomial. Therefore, $j$ can vary from 0 to 2 , enabling access to the second derivatives of the 


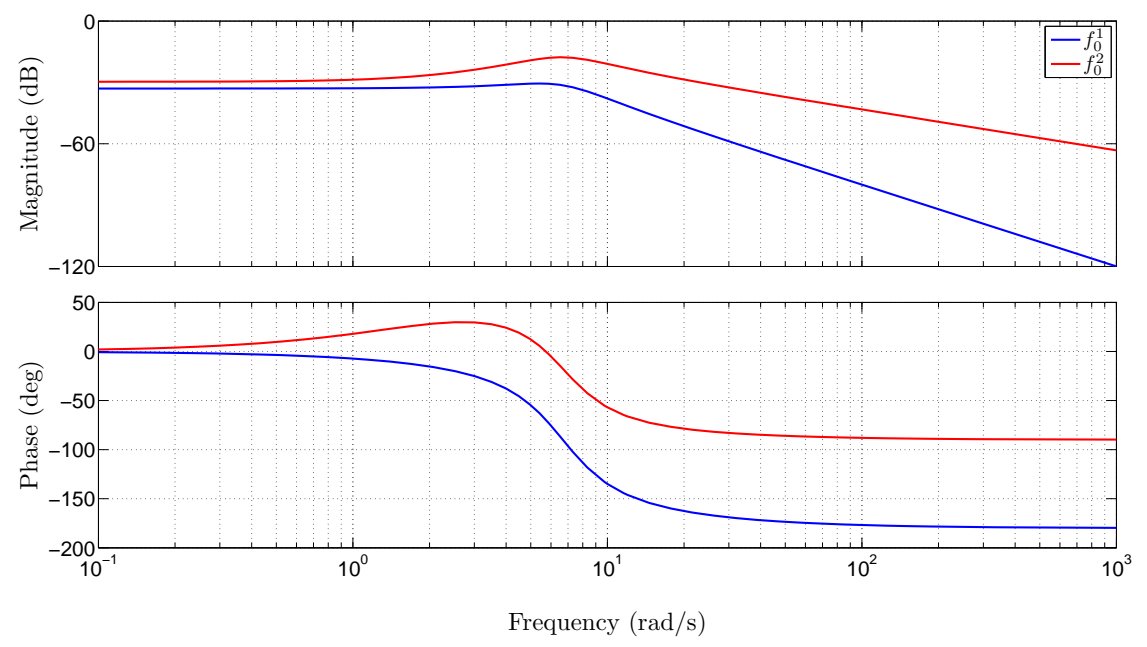

Fig. 1 Example of frequency responses - Filters $f_{0}^{1}$ (blue) and $f_{0}^{2}($ red)

measured signals while focusing on the frequency range of the system. The idea is to apply this filter on the dependent/observation variables, to obtain the derivatives, but also on the independent variables to reduce their noise and avoid any phase difference between these variables. Obviously, this is an iterative process which needs the estimated parameters of the previous iteration to compute the denominator $\Delta_{d y n}(p)$. In accordance with the SRIVC approach, the filter $f_{j}^{1}$ includes only the common dynamics of the system but the specific characteristics of each transfer; i.e. the numerators. However, for an airplane, the elevator is referred to as a rate command [19]. One can wonder if the transfer between the elevator and the pitch rate, referred to as $F_{q}$, does provide more information about the system's bandwidth than the sole denominator. Hence, it could be interesting to consider the filter:

$$
f_{j}^{2}(p)=p^{j} F_{q}(p)=p^{j} \frac{q(p)}{\Delta \delta_{e}(p)}=p^{j} \frac{k_{q}\left(p+1 / T_{\theta_{2}}\right)}{\Delta_{d y n}(p)},
$$

where $k_{q}$ and $T_{\theta_{2}}$ are respectively the constant gain of the transfer and the incidence lag [19]. In this case, $j$ can only vary between 0 and 1 in order the system to be proper. That is not a limitation because only first order time derivatives are required. Fig. 1 1 illustrates both dynamic model filters without differentiation for the aircraft described in section IV.A. As expected, $f_{0}^{2}$ has a larger gain in the bandwidth. Nonetheless, that comes at the price of a less steep low-pass filtering. The careful reader would have noticed that the SRIVC method is able to identify directly the transfer $\frac{q(p)}{\Delta \delta_{e}(p)}$. Nevertheless, the polynomials coefficients of that transfer are non-linear combinations of the aerodynamic and control derivatives to be estimated. This explains why a hybrid method is introduced in this paper.

\section{Implementation of the IV Method}

The complete iterative IV process can be described as follows:

1) Initialization. For the initial parameters, $\widehat{\boldsymbol{\theta}}^{0}$, we use the wind tunnel values or an initialization with LS estimates. The observation vector and matrix are constructed depending on the 
selected solution for the numerical differentiation as explained in section [II.C. If necessary, the input signal of the auxiliary model is filtered.

2) Iteration: repeat the following steps until convergence ( $i t$ stands for the $i t^{\text {th }}$ iteration)

(a) Simulate the auxiliary model to retrieve the noise-free signals for the instruments by using $\widehat{\boldsymbol{\theta}}^{i t-1}$.

(b) Construct the instrumental matrix using the same equations as those of the observation matrix.

(c) In the case where the pre-filtering is performed with the Butterworth filter, the practitioner can directly go to step (d). Otherwise, the instruments as well as the dependent and independent variables must be processed through a dynamic model filter $f_{j}^{1}$ or $f_{j}^{2}$, by selecting the right value of $j$ to get the derivatives.

(d) Compute the latest IV estimate of the stability and control derivatives using

$$
\widehat{\boldsymbol{\theta}}_{I V}^{i t}=\left[\frac{1}{N} \sum_{i=1}^{N} \boldsymbol{Z}^{T}\left(t_{i}, \widehat{\boldsymbol{\theta}}^{i t-1}\right) \boldsymbol{X}\left(t_{i}\right)\right]^{-1}\left[\frac{1}{N} \sum_{i=1}^{N} \boldsymbol{Z}^{T}\left(t_{i}, \widehat{\boldsymbol{\theta}}^{i t-1}\right) \boldsymbol{y}\left(t_{i}\right)\right]
$$

3) Estimated covariance. After convergence, the estimated parametric error covariance matrix of the physical parameters is computed from the following relation

$$
\boldsymbol{P}(\widehat{\boldsymbol{\theta}})=\left\{\frac{1}{N} \sum_{i=1}^{N} \boldsymbol{Z}^{T}\left(t_{i}, \widehat{\boldsymbol{\theta}}\right) \widehat{\boldsymbol{\Lambda}}^{-1} \boldsymbol{Z}\left(t_{i} \widehat{\boldsymbol{\theta}}\right)\right\}^{-1},
$$

where $\widehat{\boldsymbol{\Lambda}}$ is the $\left(n_{y} \times n_{y}\right)$ estimated covariance matrix of the filtered error $\boldsymbol{v}_{f}$ and $\widehat{\boldsymbol{\theta}}$ is the vector of estimated parameters.

It should be noticed that $(10)$ is an approximation to the real covariance formula. The underlying assumption is that the instrumental matrix has converged to the noise-free part of the observation matrix and that the residual error is a zero mean, serially uncorrelated sequence of random variables with a normal distribution ('white noise'). The convergence criterion is a test on the relative variation of the estimated parameters: $\left\|\widehat{\boldsymbol{\theta}}^{i t}-\widehat{\boldsymbol{\theta}}^{i t-1}\right\| /\left\|\widehat{\boldsymbol{\theta}}^{i t}\right\| \leq$ tol $_{\theta}$. The algorithm parameter $\operatorname{tol}_{\theta}$ must be chosen to have a good compromise between accuracy and convergence speed. In practice, choosing it between 1.0 and $5.0 \%$ appeared to be a satisfactory compromise. A criterion on the predicted outputs could also be taken into account [11]. Fig. 2] and 3 illustrate the method with the low-pass filter and the dynamic model filter respectively, by considering the SPPO model described in section II.B. In those figures, the auxiliary model's input $u$ designates the elevator deflection information that may be preprocessed to avoid the noise correlation. At first, $\Delta \delta_{e_{m}}$ is not an appropriate candidate. This point is further investigated in sections IV.B and IV.C.

\section{Results}

\section{A. Test Aircraft and Flight Conditions}

For the numerical evaluation of the IV approach, the Generic Transport Model (GTM) is considered. This is a 5.5 percent dynamically-scaled model of a generic commercial twin-engine jet transport airplane. Further information on the GTM and associated flight tests can be found in 


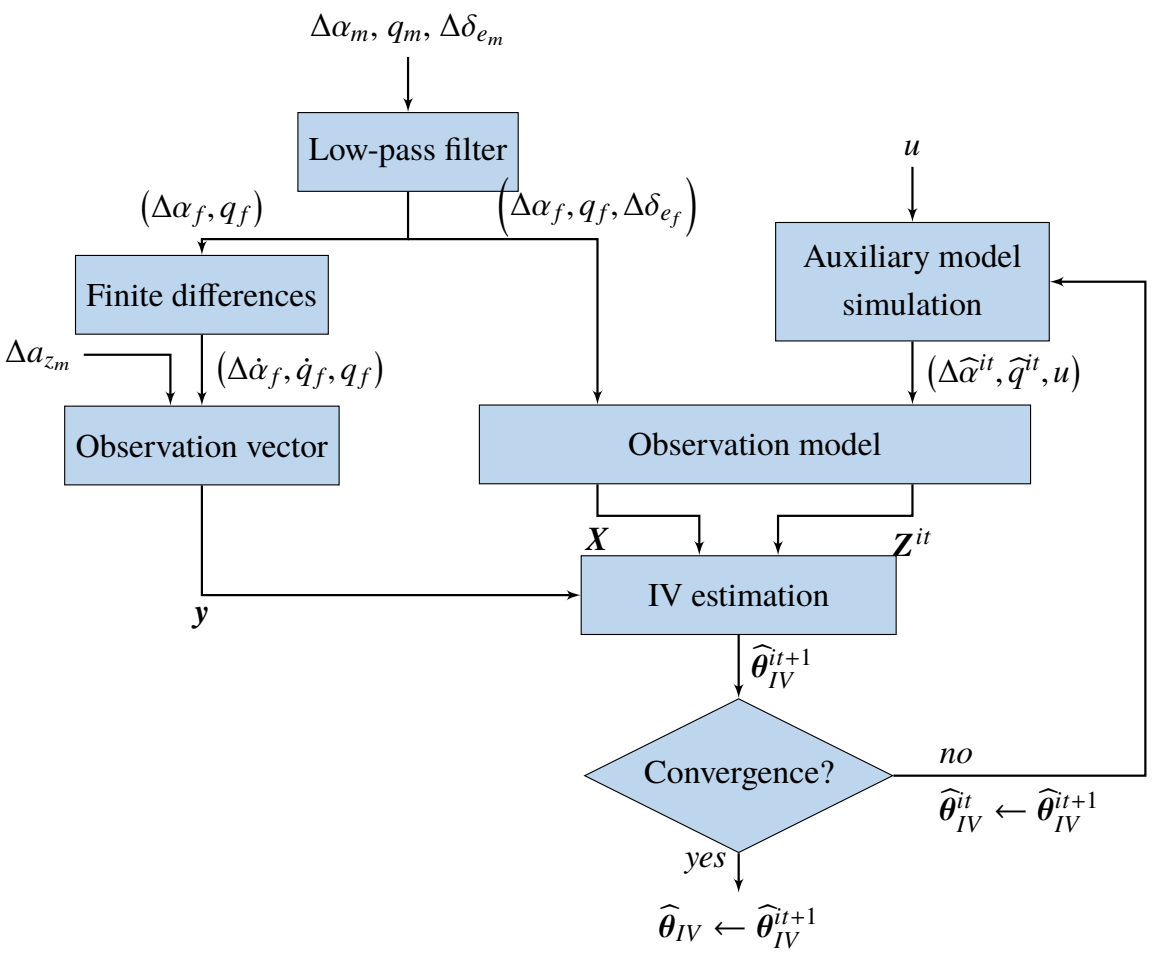

Fig. 2 Block diagram of the IV approach with low-pass filtering and SPPO model

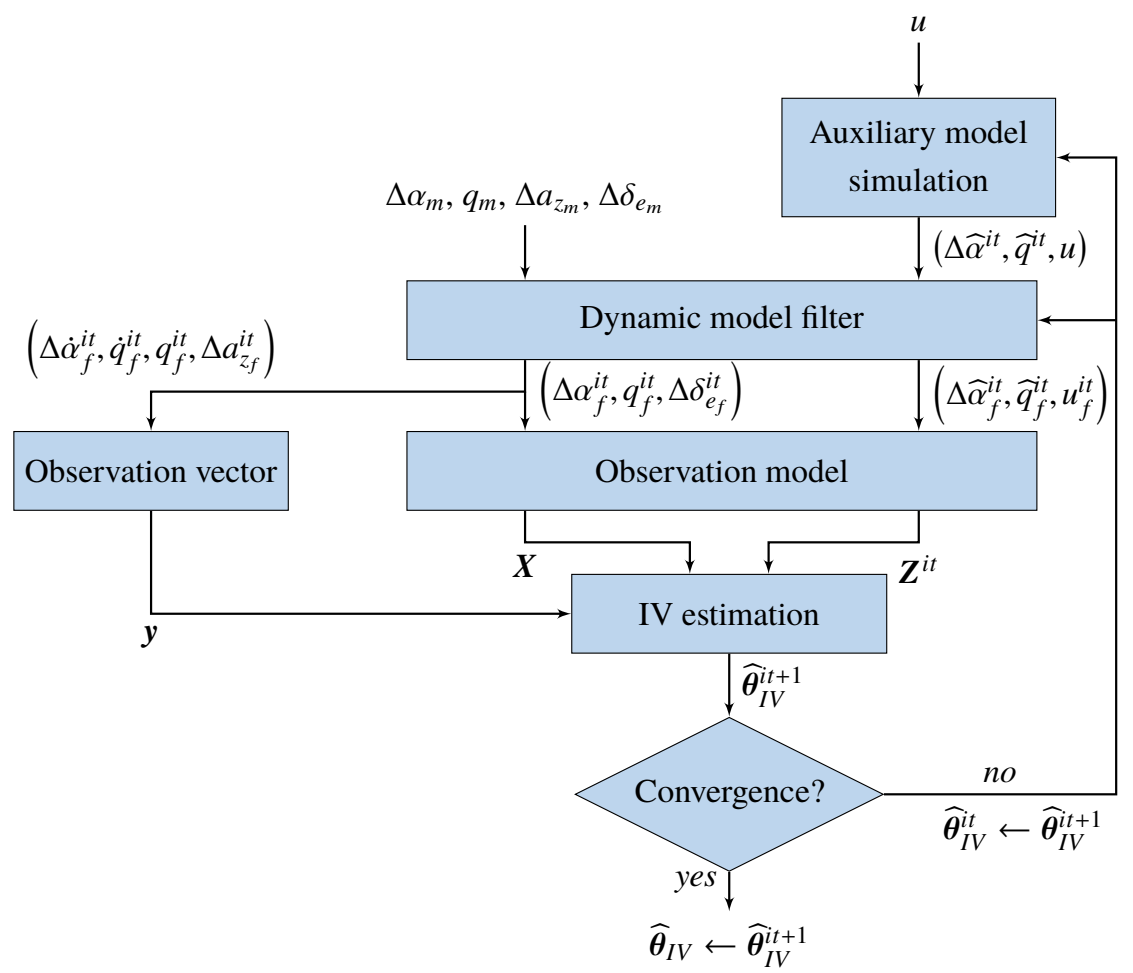

Fig. 3 Block diagram of the IV approach with dynamic model filtering and SPPO model 
Refs. [5, 20]. The nonlinear simulation is based on a database coming from wind tunnel data. For the longitudinal control, the GTM has four surfaces: left and right inboard and outboard elevators. Those elevators are fused as single elevator surface accordingly to $\delta_{e}=\frac{1}{4}\left(\delta_{e}^{l o}+\delta_{e}^{l i}+\delta_{e}^{r i}+\delta_{e}^{r o}\right)$.

The flight conditions of the simulation are $E A S=75 \mathrm{kt}, \alpha=5.56 \mathrm{deg}, h=7000 \mathrm{ft}$ and $\delta_{e}=0.10 \mathrm{deg}$. The GTM simulator has the advantage of providing a Dryden turbulence model with 3 levels of intensity: light, moderate and severe, based on values provided in Ref. [21]. In addition, the simulator includes a modeling of the sensors errors: noise and biases. All variances are the original values provided in the GTM* The data were simulated at $200 \mathrm{~Hz}$ rate, but the identification is performed from data down-sampled at $50 \mathrm{~Hz}$ for the sake of realism. A doublet input [13] was used to excite the elevators during straight and level trimmed flight. The doublet has a time step of 0.5 and an amplitude of $2 \mathrm{deg}$. The same input sequence was used in each set of data.

\section{B. Influence of the input signal}

In a first time, to validate the IV method, a comparison of the input signals is performed. The idea is to evaluate the sensitivity of the method to the input signal of the auxiliary model. As explained in section III.A, the goal is to ensure the instrument exogeneity. In practice, the observation matrix is built with the low-pass filter, as described in section III.C and by Fig. 2. The filter is a $4^{\text {th }}$ order Butterworth one with a cut-off frequency at $5 \mathrm{~Hz}$. Three different inputs are considered:

- $\Delta \delta_{e_{f}}$ : the measured surface deflection processed by the same Butterworth filter as the other measurements to build the observation matrix;

- $\Delta \delta_{e}^{r}$ : the reference surface trajectory sent to actuators;

- $\Delta \widehat{\delta}_{e}$ : the estimated surface deflection via the identification of the actuators.

The filtered signal $\Delta \delta_{e_{f}}$ is the most straightforward solution because the filtering process must be applied to the other measurements. In theory, the reference trajectory $\Delta \delta_{e}^{r}$ is a convenient solution since it is perfectly know (noise-free) and correlated with $\Delta \delta_{e_{m}}$. However, if the actuator dynamics is too slow, the generated instruments may be not correlated enough with the experimental signals. This is why the identification of the actuators is considered. In practice, the transfer between $\Delta \delta_{e}^{r}$ and $\Delta \delta_{e}$ is identified as a continuous-time first order system thanks to the SRIVC method available in CONtinuous-Time System IDentification toolbox (CONTSID)

The techniques are evaluated with added sensors noises in the case of light turbulence. 300 Monte Carlo Simulations (MCS) with independent noise sequences are run. The 'true' values of the parameters come from a linearization of the database about the trim conditions. All the IV variants are initialized with the LS estimation. It should be noticed that all the signals are filtered through the Butterworth before being employed by the LS method. The method is therefore in favorable position with regard to the turbulence aspects because the frequency range was selected in line with the input spectrum. Table 1 provides the mean results for stability and control derivatives of the 300 MCS as well as the standard deviations, the relative errors and the average numbers of iterations (Av. iter.) for IV convergence. Table 1 also gives the mean modal parameters: the natural frequency $\omega_{n}$ and the damping factor $\zeta$. The 'true' modal parameters come from the SPPO reduced order model. It appears that the LS method has major difficulties to estimate the parameters of the lift coefficient. In the opposite, the IV variants provide more accurate estimations of the aerodynamic and control parameters, although there is large uncertainty with regard to $C_{L q}$. That is likely due to

*This model can be downloaded via https://github.com/nasa/GTM_DesignSim.

$\dagger$ This toolbox can be downloaded via http://www. contsid.cran.univ-lorraine. fr/. 
Table 1 Input influence - Light turbulence - 300 MCS - Mean estimated parameters and their standard deviations

\begin{tabular}{|c|c|c|c|c|c|}
\hline$\theta$ & True Value & LS & $\operatorname{IV}\left(\Delta \delta_{e_{f}}\right)$ & $\mathrm{IV}\left(\Delta \delta_{e}^{r}\right)$ & $\operatorname{IV}\left(\Delta \widehat{\delta}_{e}\right)$ \\
\hline$C_{L \alpha}$ & 4.793 & $4.101(0.418)$ & $4.713(0.196)$ & $4.646(0.213)$ & $4.697(0.197)$ \\
\hline$C_{L q}$ & 30.79 & $88.46(45.04)$ & $34.48(16.41)$ & $29.50(13.17)$ & 32.68 (14.62) \\
\hline$C_{L \delta_{e}}$ & 0.480 & $1.060(0.702)$ & $0.456(0.356)$ & $0.315(0.258)$ & $0.407(0.302)$ \\
\hline$C_{m \alpha}$ & -1.789 & $-1.569(0.066)$ & $-1.594(0.054)$ & $-1.325(0.058)$ & $-1.523(0.053)$ \\
\hline$C_{m q}$ & -41.41 & $-38.118(3.465)$ & $-36.61(2.360)$ & $-21.04(2.517)$ & $-32.52(2.117)$ \\
\hline \multirow[t]{3}{*}{$C_{m \delta_{e}}$} & -1.787 & $-1.758(0.087)$ & $-1.739(0.065)$ & $-1.293(0.055)$ & $-1.626(0.056)$ \\
\hline & $\operatorname{RelErr}(\widehat{\theta})$ & $111 \%$ & $11.7 \%$ & $39.4 \%$ & $17.5 \%$ \\
\hline & Av. iter. & - & 3.96 & 5.62 & 3.84 \\
\hline$\omega_{n}$ & 6.632 & $5.928(0.245)$ & $6.242(0.130)$ & $5.578(0.125)$ & $6.076(0.124)$ \\
\hline \multirow[t]{3}{*}{$\zeta$} & 0.388 & $0.386(0.024)$ & $0.379(0.012)$ & $0.321(0.014)$ & $0.365(0.011)$ \\
\hline & $\operatorname{RelErr}\left(\widehat{\omega}_{n}\right)$ & $10.6 \%$ & $5.89 \%$ & $15.9 \%$ & $8.36 \%$ \\
\hline & $\operatorname{RelErr}(\widehat{\zeta})$ & $0.53 \%$ & $2.26 \%$ & $17.2 \%$ & $5.98 \%$ \\
\hline
\end{tabular}

a lack of sensitivity for this parameter, which has already been observed in previous works [17]. The variant based on the reference trajectory $\Delta \delta_{e}^{r}$ seems less accurate than the two others and has more difficulties to converge. Finally, the variant $\Delta \delta_{e_{f}}$ gives more relevant estimated parameters than the variant based on $\Delta \widehat{\delta}_{e}$. That can be explained by the relatively low sampling frequency for the continuous-time identification of the actuator. This assumption was validated with a dedicated test at $200 \mathrm{~Hz}$, not shown for the sake of clarity.

As a result of this first investigation related to the input of the auxiliary model, it comes out that:

- The LS method is in default under turbulent conditions as predicted;

- The generation of the instruments using the reference surface trajectory $\delta_{e}^{r}$ is not appropriate. The time constant of the actuator is indeed too large that leads to instruments not correlated enough with the observation matrix. This lack of correlation is referred to as weak instruments [14].

- The continuous-time identification of the actuator dynamics is not enough accurate at the current sampling frequency to build instruments correlated enough;

- The low-pass filtering is the most effective solution to remove the noise and ensure the exogeinity.

Thereafter, as long as the observation is built with the low-pass filtering, the input of auxiliary model is always the measured surface deflection filtered by the low-pass filter. In other words, we have $u=\Delta \delta_{e_{f}}$ in Fig. 2 ,

\section{Influence of the Filtering Technique}

This part is devoted to the investigation of the filtering through the dynamic model. The simulation parameters are still a light turbulence and $300 \mathrm{MCS}$. The goal is to evaluate the relevance of the dynamic model filtering by comparing the $f^{1}$ and $f^{2}$ solutions developed in section III.C. 
With respect to Fig. 3, the input of the auxiliary model is the measured elevator deflection; i.e. $u=\Delta \delta_{e_{m}}$. One can wonder why there is no preprocessing to avoid noise correlation. In fact, all the simulated signals included the input are filtered through the dynamic model. Since the observation model is linear with respect to the parameters and the states, that filtering process can commute with the simulation, which can also be seen as a filtering operation. For example, by considering the transfer $F_{q}$ at iteration $i t$ with the filter $f^{1}$, it comes out

$$
\begin{aligned}
f^{1}\left(p, \widehat{\theta}^{i t}\right) \cdot \widehat{q}^{i t}(t) & =f^{1}\left(p, \widehat{\theta}^{i t}\right) \cdot F_{q}\left(p, \widehat{\theta}^{i t}\right) \cdot \Delta \delta_{e_{m}}(t) \\
\leftrightarrow \quad \widehat{q}_{f}^{i t}(t) & =F_{q}\left(p, \widehat{\theta}^{i t}\right) \cdot f^{1}\left(p, \widehat{\theta}^{i t}\right) \cdot \Delta \delta_{e_{m}}(t)=F_{q}\left(p, \widehat{\theta}^{i t}\right) \cdot \Delta \delta_{e_{f^{1}}}^{i t}(t)
\end{aligned}
$$

Table 2 depicts the results of the MCS for the stability and control derivatives. The $f^{2}$ solution performs as well as the low-pass filtering studied in the previous part, $\Delta \delta_{e_{f}}$, with respect to the aerodynamic parameters. Nonetheless, the damping ratio of the $f^{2}$ solution is slightly more accurate than the one of the $\Delta \delta_{e_{f}}$ method. On the other hand, the $f^{1}$ solution provides more accurate mean estimations, irrespective of the considered parameter, although it requires in average a little more of iterations. Consequently, the dynamic model filtering appears to be an elegant solution to deal with the turbulent noise and the noisy signals differentiation. Furthermore, it seems that their is an advantage to consider the $f^{1}$ solution inspired from the original SRIVC method. Fig. 4 depicts the outputs time histories for the LS and IV ( $f^{1}$ variant) methods for the last iteration of the MCS. The IV $\left(\Delta \delta_{e_{f}}\right)$ and IV $\left(f^{2}\right)$ results are not presented as they do not give more insight. As expected, the turbulence effects are especially visible on the first output $y_{1}=\dot{\alpha}-q$ because it contains an airflow measurement. Despite the discrepancies in the stability parameters in Table 2, the outputs do not show large differences. There is only a small discrepancy on $a_{z}$ between 3 and 3.5 seconds. This similarity between the estimated outputs reflects a lack of sensitivity for some parameters, like $C_{L q}$ for instance.

\section{Instrumental Variable for Parameter Estimation in Moderate Turbulence}

In this last experimental part, the three previous IV variants are considered along with a moderate turbulence and a non-zero constant wind. At each MCS, the wind speed is generated from a normal distribution with zero mean and $30 \mathrm{kt}$ of standard deviation. The goal of this experiment is to test the limits of the methods. Since it brings another random parameter in the problem, the number of MCS is increased to 600 .

The mean estimated parameters and their standard deviations, as well as the corresponding modal parameters, are given by Table 3. By looking at the aerodynamic parameters, it appears that the LS method provides an inaccurate estimation, although this is not clear by looking at the damping ratio. In general, the damping ratio is less impacted than the natural frequency, irrespective of the method or the turbulence level. The mean estimated parameters of the IV methods are not too impacted by the wind and the rise of the turbulence level. Nonetheless, those phenomena lead to larger standard deviations and the algorithms needed more iterations to convergence. In this case, the Butterworth and $f^{2}$ solutions perform equivalently by looking at the aerodynamic parameters. Here also, the $f^{1}$ appears to be more competitive. 
Table 2 Filter influence - Light turbulence - 300 MCS - Mean estimated parameters and their standard deviations

\begin{tabular}{|c|c|c|c|c|c|}
\hline$\theta$ & True Value & LS & $\operatorname{IV}\left(\Delta \delta_{e_{f}}\right)$ & $\operatorname{IV}\left(f^{1}\right)$ & $\operatorname{IV}\left(f^{2}\right)$ \\
\hline$C_{L \alpha}$ & 4.793 & $4.101(0.418)$ & $4.713(0.196)$ & $4.708(0.176)$ & $4.707(0.172)$ \\
\hline$C_{L q}$ & 30.79 & 88.46 (45.04) & $34.48(16.41)$ & $33.94(19.4)$ & $35.28(12.26)$ \\
\hline$C_{L \delta_{e}}$ & 0.480 & $1.060(0.702)$ & $0.456(0.356)$ & $0.443(0.455)$ & $0.479(0.252)$ \\
\hline$C_{m \alpha}$ & -1.789 & $-1.569(0.066)$ & $-1.594(0.054)$ & $-1.613(0.052)$ & $-1.605(0.052)$ \\
\hline$C_{m q}$ & -41.41 & $-38.118(3.465)$ & $-36.61(2.360)$ & $-38.12(2.374)$ & $-37.54(2.386)$ \\
\hline \multirow[t]{3}{*}{$C_{m \delta_{e}}$} & -1.787 & $-1.758(0.087)$ & $-1.739(0.065)$ & $-1.788(0.067)$ & $-1.738(0.066)$ \\
\hline & $\operatorname{RelErr}(\widehat{\theta})$ & $111 \%$ & $11.7 \%$ & $8.79 \%$ & $11.4 \%$ \\
\hline & Av. iter. & - & 3.96 & 4.55 & 4.23 \\
\hline$\omega_{n}$ & 6.632 & $5.928(0.245)$ & $6.242(0.130)$ & $6.293(0.133)$ & $6.267(0.116)$ \\
\hline \multirow[t]{3}{*}{$\zeta$} & 0.388 & $0.386(0.024)$ & $0.379(0.012)$ & $0.384(0.013)$ & $0.383(0.012)$ \\
\hline & $\operatorname{RelErr}\left(\widehat{\omega}_{n}\right)$ & $10.6 \%$ & $5.89 \%$ & $5.12 \%$ & $5.51 \%$ \\
\hline & $\operatorname{RelErr}(\widehat{\zeta})$ & $0.53 \%$ & $2.26 \%$ & $0.86 \%$ & $1.34 \%$ \\
\hline
\end{tabular}
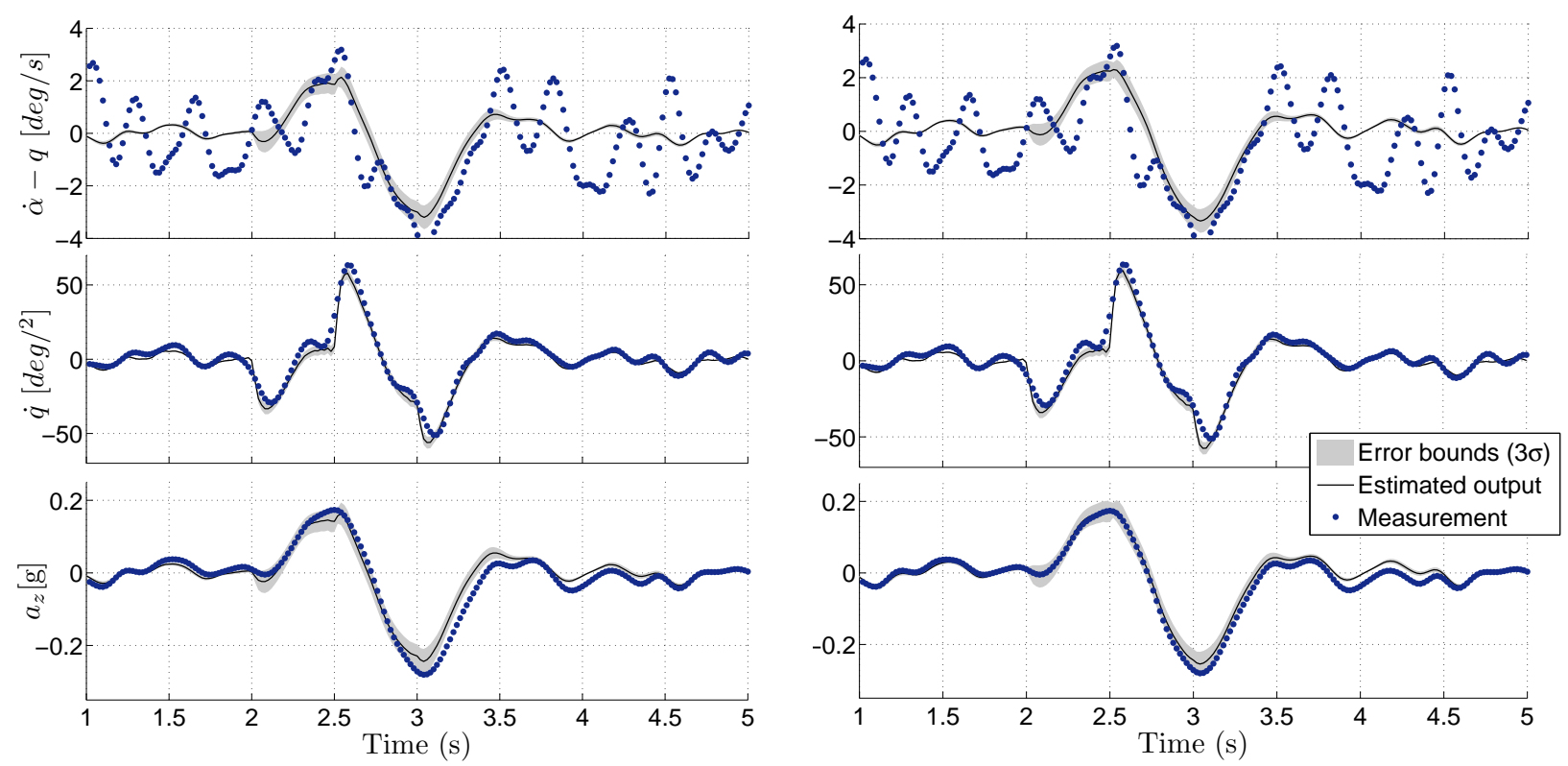

Fig. 4 Light turbulence - Estimated (black line) and measured (blue dot) outputs - LS (left) and $\mathrm{IV} / f^{1}$ (right) methods 
Table 3 Moderate turbulence - 600 MCS - Mean estimated parameters and their standard deviations

\begin{tabular}{|c|c|c|c|c|c|}
\hline$\theta$ & True Value & LS & $\operatorname{IV}\left(\Delta \delta_{e_{f}}\right)$ & $\operatorname{IV}\left(f^{1}\right)$ & $\operatorname{IV}\left(f^{2}\right)$ \\
\hline$C_{L \alpha}$ & 4.793 & $3.546(0.653)$ & $4.727(0.394)$ & $4.726(0.381)$ & $4.709(0.362)$ \\
\hline$C_{L q}$ & 30.79 & $106(57.99)$ & $32.59(35.28)$ & $32.16(46.4)$ & $34.33(30.21)$ \\
\hline$C_{L \delta_{e}}$ & 0.480 & $1.070(0.943)$ & $0.404(0.754)$ & $0.401(1.048)$ & $0.454(0.615)$ \\
\hline$C_{m \alpha}$ & -1.789 & $-1.583(0.075)$ & $-1.597(0.106)$ & $-1.617(0.102)$ & $-1.608(0.102)$ \\
\hline$C_{m q}$ & -41.41 & $-41.63(3.587)$ & $-36.34(3.696)$ & $-37.93(4.382)$ & $-37.58(4.548)$ \\
\hline \multirow[t]{3}{*}{$C_{m \delta_{e}}$} & -1.787 & $-1.826(0.091)$ & $-1.733(0.103)$ & $-1.787(0.118)$ & $-1.741(0.126)$ \\
\hline & $\operatorname{RelErr}(\widehat{\theta})$ & $146 \%$ & $10.4 \%$ & $7.21 \%$ & $10.0 \%$ \\
\hline & Av. iter. & - & 4.47 & 5.59 & 5.18 \\
\hline$\omega_{n}$ & 6.632 & $5.846(0.365)$ & $6.248(0.266)$ & $6.302(0.295)$ & $6.273(0.245)$ \\
\hline \multirow[t]{3}{*}{$\zeta$} & 0.388 & $0.392(0.028)$ & $0.378(0.020)$ & $0.384(0.025)$ & $0.383(0.02)$ \\
\hline & $\operatorname{RelErr}\left(\widehat{\omega}_{n}\right)$ & $11.9 \%$ & $5.79 \%$ & $4.98 \%$ & $5.41 \%$ \\
\hline & $\operatorname{RelErr}(\widehat{\zeta})$ & $1.16 \%$ & $2.55 \%$ & $1.04 \%$ & $1.35 \%$ \\
\hline
\end{tabular}

\section{Conclusion}

A relevant Instrumental Variable (IV) approach for aircraft identification is introduced in this paper. The set of instruments is generated with the simulation of the dynamic model assuming the same structure as the identified model, the same excitation signal and using the estimated parameters of the previous iteration. The initialization is successfully performed with the leastsquares estimation. The rapid converge makes the calculations tractable. Two filtering strategies are considered to estimate the signal derivatives necessary to build the observation vector. The first one requires the selection of the frequency range by the user, whereas the second one automatically selects this range via the dynamic model identified at the previous iteration. The difficulty of assuring the input exogeneity is tackled via different dedicated strategies.

A validation is carried out on the Generic Transport Model flying in turbulent air. The following features were checked:

- The IV approach is more robust to turbulence than the Least-Square approach;

- The IV approach has a rapid convergence;

- The instruments can be generated with a filtered elevator deflection;

- A filtering by the dynamic model, inspired by the Simplified Refined IV for Continuous-time systems, allows an automatic identification process.

Future work concerns the validation of the approach on more complex models and by using experimental signals.

\section{Acknowledgments}

I am very grateful to Dr. Alex Janot for converting me to the IV approach to electro-mechanical systems. I have also benefited from discussion with Prof. Peter C. Young, who has contributed so much to the continuous-time systems identification via the IV approach. 


\section{References}

[1] Greenberg, H., "A survey of methods for determining stability parameters of an airplane from dynamic flight measurements," Tech. Rep. 2340, NACA, 1951.

[2] Klein, V., "Identification evaluation methods," AGARD lecture series, Vol. 104, 1979.

[3] Jonkers, H. L., "Application of the Kalman filter to flight path reconstruction from flight test data including estimation of instrumental bias error corrections," Delft University of Technology, Department of Aerospace Engineering, Report VTH-162, 1976.

[4] Morelli, E., "Practical aspects of the equation-error method for aircraft parameter estimation," AIAA Atmospheric Flight Mechanics Conference and Exhibit, 2006, p. 6144. doi:10.2514/6.2006-6144.

[5] Morelli, E., and Cunningham, K., "Aircraft dynamic modeling in turbulence," AIAA Atmospheric Flight Mechanics Conference, 2012, p. 4650. doi:10.2514/6.2012-4650.

[6] Maine, R. E., and Iliff, K. W., "Formulation and implementation of a practical algorithm for parameter estimation with process and measurement noise," SIAM journal on applied mathematics, Vol. 41, No. 3 , 1981, pp. 558-579.

[7] Reiersøl, O., "Confluence analysis by means of lag moments and other methods of confluence analysis," Econometrica: Journal of the Econometric Society, 1941, pp. 1-24.

[8] Wong, K., and Polak, E., "Identification of linear discrete time systems using the instrumental variable method," IEEE Transactions on Automatic Control, Vol. 12, No. 6, 1967, pp. 707-718.

[9] Söderström, T., Stoica, P., and Stoica, P., Instrumental variable methods for system identification, Lectures Notes in Control and Information Sciences, Springer-Verlag, Berlin, 1983.

[10] Gilson, M., Garnier, H., Young, P. C., and Van den Hof, P. M., "Optimal instrumental variable method for closed-loop identification," IET control theory \& applications, Vol. 5, No. 10, 2011, pp. 1147-1154.

[11] Janot, A., Vandanjon, P.-O., and Gautier, M., "A generic instrumental variable approach for industrial robot identification," IEEE Transactions on Control Systems Technology, Vol. 22, No. 1, 2014, pp. 132-145.

[12] Larsson, R., and Enqvist, M., "Sequential Aerodynamic Model Parameter Identification," IFAC Proceedings Volumes, Vol. 45, No. 16, 2012, pp. 1413 - 1418. 16th IFAC Symposium on System Identification.

[13] Klein, V., and Morelli, E. A., Aircraft system identification: theory and practice, American Institute of Aeronautics and Astronautics Reston, Va, USA, 2006.

[14] Wooldridge, J. M., Introductory econometrics: A modern approach, Nelson Education, 2015.

[15] Young, P. C., Recursive estimation and time-series analysis: an introduction, Springer Science \& Business Media, 2012.

[16] Greenberg, H., "Determination of stability derivatives from flight data," Journal of the Aeronautical Sciences, Vol. 16, No. 1, 1949, p. 62. 
[17] Brunot, M., "Comparison of Numerical Differentiation Techniques for Aircraft Identification," Journal of Aerospace Engineering, Vol. 32, No. 5, 2019, p. 06019002.

[18] Aljanaideh, K. F., and Bernstein, D. S., "Initial Conditions in Time-and Frequency-Domain System Identification: Implications of the Shift Operator Versus the $\mathrm{Z}$ and Discrete Fourier Transforms," IEEE Control Systems, Vol. 38, No. 2, 2018, pp. 80-93.

[19] Cook, M. V., Flight dynamics principles: a linear systems approach to aircraft stability and control, Butterworth-Heinemann, 2012.

[20] Jordan, T., Langford, W., Belcastro, C., Foster, J., Shah, G., Howland, G., and Kidd, R., "Development of a dynamically scaled generic transport model testbed for flight research experiments," Tech. rep., NASA, 2004.

[21] Johnson, D. L., "Terrestrial environment (climatic) criteria guidelines for use in aerospace vehicle development, 1993 revision,” Tech. Rep. TM 4511, NASA, August 1993. 